International Journal of Pure and Applied Mathematics

Volume 115 No. 4 2017, 813-820

ISSN: 1311-8080 (printed version); ISSN: 1314-3395 (on-line version)

url: http://www.ijpam.eu

doi: 10.12732/ijpam.v115i4.14

\title{
ON GENERALIZED HERMITE-HADAMARD TYPE INEQUALITIES FOR GENERALIZED HARMONIC INVEX FUNCTIONS
}

\author{
S.N. Mishra ${ }^{1}$, P.K. Das ${ }^{2} \S$, G.C. Nayak $^{3}$ \\ ${ }^{1,2}$ Department of Mathematics \\ School of Applied Sciences \\ KIIT University \\ Bhubaneswar, 751024, INDIA \\ Department of Mathematics \\ Govt. Junior College \\ Phulbani, Kandhamala, 762001, INDIA
}

\section{Abstract:}

AMS Subject Classification: 26A51, 26B25, 52A41, 26D15

Key Words: Hermite-Hadamard inequality, Hermite-Hadamard type inequality, invariant harmonic convex set, invariant harmonic convex function

\section{Introduction}

Let $X$ be a topological vector space. Let $K \subset X \backslash\{0\}$ be a set satisfying the following conditions. For $x, y \in K$, let $I[y, x]$ be a path joining $y$ and $x$ contained in $K$ and the map $\gamma_{x y}:[0,1] \rightarrow I[y, x]$ be continuous. Let $\eta: K \times K \rightarrow X$ be any vector valued mapping. The set $K$ has the harmonic invex combination (HIC) property with respect to the direction $\eta$ if the followings are satisfied:

$(P 1) y+t \eta(x, y) \in K$ for all $t \in[0,1], x, y \in K$,

Received: $\quad$ March 6, 2017

Revised: $\quad$ May 6, 2017

Published: August 8, 2017

$\S$ Correspondence author (c) 2017 Academic Publications, Ltd. url: www.acadpubl.eu 
$(P 2) y+t \eta(x, y)=\left\{\begin{array}{ll}y, & \text { if } t=0 ; \\ x, & \text { if } t=1\end{array}\right.$ iff $y+t \eta(x, y)=\frac{x+y}{2}$ for $t=\frac{1}{2}$

(P3) for any $z \in I[y, x] \subset K$, we have

$$
z=y+t \eta(x, y)=x+(1-t) \eta(x, y)
$$

(P4) $\frac{x y}{y+t \eta(x, y)} \in I[y, x]$ for all $x, y \in K$.

Let $X=\mathbb{R}$ and for any $\eta: K \times K \rightarrow X, h: K \times K \times[0,1] \rightarrow \mathbb{R}_{+} K=K_{\eta}=$ $[a, b] \subset X \backslash\{0\}$ be a set with HIC properties $P_{1}$ to $P_{4}$ in the direction $\eta$. Then the inequality

$$
f\left(\frac{2 a b}{a+b}\right) \leq \frac{a b \bar{h}(x, y)}{\eta(a, b)} \int_{a}^{b} \frac{f(x)}{x^{2}} d x \leq[\bar{h}(x, y)]^{2} \frac{f(a)+f(b)}{2}
$$

hold if the mapping $f: K \rightarrow \mathbb{R}$ is a harmonically invex function with respect to $\eta$ associated with $h$ on $K_{\eta}$ defined in equation (4).

Remark 1. The inequality (1) coincides with the inequality $(2)$ if $\eta(a, b)=$ $b-a$ and $\bar{h}(x, y)=1$.

\section{Preliminaries}

Hermite and Hadamard have studied an integral inequality associated with a convex function as follows: Let $K \subset \mathbb{R}$ be a convex set and $f: K \rightarrow \mathbb{R}$ be a convex function. Then for any interval $[a, b] \subset K$, the inequality

$$
f\left(\frac{a+b}{2}\right) \leq \frac{1}{b-a} \int_{a}^{b} f(x) d x \leq \frac{f(a)+f(b)}{2}
$$

hold. This integral inequality is known as Hermite and Hadamard inequality. In 2014, I. Insan [3] has introduced the concept of harmonically convex set and harmonically convex functions and has studied the Hermite-Hadamard type inequalities for harmonically convex functions as an extension work of HermiteHadamard inequalities. We recall the concepts of harmonic sets and harmonic convex function introduced by Insan [3].

Definition 2. Let $K \subset \mathbb{R} \backslash\{0\}$ be any set and $f: K \rightarrow \mathbb{R}$ be any map.

1. $K$ is said to be harmonic convex set if

$$
\frac{x y}{t x+(1-t) y} \in K
$$

for all $x, y \in K$ and $t \in[0,1]$, 
2. $f$ is said to be harmonically convex function on the harmonically convex set $K$ if

$$
f\left(\frac{x y}{t x+(1-t) y}\right) \leq t f(y)+(1-t) f(x)
$$

for all $x, y \in K$.

We recall the Hermite-Hadamard type inequalities for harmonically convex functions studied by Insan [3]. Let $L[a, b]$ be the set of integrable functions in the interval $[a, b]$.

Theorem 3. Let $f: K \subset \mathbb{R} \backslash\{0\} \rightarrow \mathbb{R}$ be a harmonically convex function and $a, b \in K$ with $a<b$. If $f \in L[a, b]$, then the following inequalities hold

$$
f\left(\frac{2 a b}{a+b}\right) \leq \frac{a b}{b-a} \int_{a}^{b} \frac{f(x)}{x^{2}} d x \leq \frac{f(a)+f(b)}{2} .
$$

The above inequalities are sharp.

Theorem 4. Let $f: K \subset \mathbb{R} \backslash\{0\} \rightarrow \mathbb{R}$ be a differentiable function on $\operatorname{Int}(K)$, the interior of $K$ and $a, b \in K$ with $a<b$. If $f^{\prime} \in L[a, b]$, then the following inequalities hold

$$
\begin{aligned}
& \frac{f(a)+f(b)}{2}-\frac{a b}{b-a} \int_{a}^{b} \frac{f(x)}{x^{2}} d x \\
& =\frac{a b(b-a)}{2} \int_{0}^{1} \frac{1-2 t}{(t b+(1-t) a)^{2}} f^{\prime}\left(\frac{a b}{t b+(1-t) a}\right) d t .
\end{aligned}
$$

\section{Main Results}

In this section, we have extended the results (2) and (3) under certain conditions. For our need, we make the definition of invariant harmonically convex function in the IHC set $K$.

Definition 5. Let $K \subset \mathbb{R} \backslash\{0\}$ be any set and $f: K \rightarrow \mathbb{R}$ be any map.

1. $K$ is said to be harmonic invex (HI) set given in the direction $\eta \in \mathbb{R} \backslash 0$ if $K$ has the $H I C$ properties $P_{1}$ to $P_{4}$.

2. $f$ is said to be harmonically invex (HI) function associated with $h$ on the $H I$ set $K$ if there exists a vector function $\eta: K \times K \rightarrow X$ and $h: K \times K \times[0,1] \rightarrow \mathbb{R}_{+}=[0, \infty]$ satisfying

$$
h(x, y, t)=t \bar{h}(x, y)
$$


such that

$$
f\left(\frac{x y}{y+t \eta(x, y)}\right) \leq h(x, y, t) f(y)+(1-h(x, y, t)) f(x)
$$

for all $x, y \in K, t \in[0,1]$.

For existence of the result (1), we show the following result.

Lemma 6. For $\eta: K \times K \rightarrow X$, let $K=K_{\eta} \subset \mathbb{R} \backslash\{0\}$ be a HI set. Let $f: K \rightarrow \mathbb{R}$ be a $H I$ function on $\operatorname{Int}(K)$ with respect to the direction $\eta \in \mathbb{R} \backslash\{0\}$ associated with $h$, then for all $x, y \in K$, the following inequality hold

$$
f\left(\frac{2 x y}{x+y}\right) \leq \frac{\bar{h}(x, y)}{2}(f(x)+f(y)) .
$$

Proof. By invexity properties of $f$, we have

$$
f\left(\frac{x y}{y+t \eta(x, y)}\right) \leq h(x, y, t) f(y)+(1-h(x, y, t)) f(x)
$$

for all $x, y \in K, t \in[0,1]$. At $t=\frac{1}{2}$, we have

$$
f\left(\frac{2 x y}{2 y+\eta(x, y)}\right) \leq \bar{h}(x, y) \frac{f(x)+f(y)}{2}
$$

for all $x, y \in K$. Since $x=y+\eta(x, y)$, we have

$$
f\left(\frac{2 x y}{x+y}\right) \leq \bar{h}(x, y) \frac{f(x)+f(y)}{2}
$$

for all $x, y \in K$. This completes the proof.

Theorem 7. Let $\eta: K \times K \rightarrow X$ be a vector valued antisymmetric (i.e., $\eta(x, y)+\eta(y, x)=0$ for all $x, y \in K)$ map and $K=K_{\eta} \subset \mathbb{R} \backslash\{0\}$ be a HI set. Let $a<b \in K$. Let $f: K \rightarrow \mathbb{R}$ be a $H I$ function on $\operatorname{Int}(K)$ with respect to the direction $v \in \mathbb{R} \backslash\{0\}$ associated with $h$. Let $f \in L[a, b]$, then the following inequality hold:

$$
f\left(\frac{2 a b}{a+b}\right) \leq \frac{a b \bar{h}(x, y)}{\eta(a, b)} \int_{a}^{b} \frac{f(x)}{x^{2}} d x \leq[\bar{h}(x, y)]^{2} \frac{f(a)+f(b)}{2}
$$


Proof. In view of Lemma 6, we have

$$
f\left(\frac{2 x y}{x+y}\right) \leq \bar{h}(x, y) \frac{f(x)+f(y)}{2}
$$

for all $x, y \in K$, implying

$$
f\left(\frac{2 a b}{a+b}\right) \leq \bar{h}(a, b) \frac{f(a)+f(b)}{2}
$$

for $a, b \in K$ with $a<b$. Assuming $x=\frac{a b}{b+t \eta(a, b)}$ and $y=\frac{a b}{a+t \eta(b, a)}$, we have

$$
\frac{2 x y}{x+y}=\frac{a b}{a+b+t(\eta(a, b)+\eta(b, a))}=\frac{a b}{a+b} \text {. }
$$

If $s=\frac{\alpha \beta}{\beta+t z}$ where $t \in[0,1]$ and $\beta=\alpha+z$. As $t: 0 \rightarrow 1$,

$$
s: \alpha \rightarrow \frac{\alpha \beta}{\beta+z}=\frac{\alpha \beta}{\alpha}=\beta .
$$

Again

$$
t=\frac{1}{z}\left(\frac{\alpha \beta}{s}-\beta\right) \Rightarrow d t=-\frac{\alpha \beta}{z s^{2}} d s .
$$

Thus

$$
\int_{0}^{1} f\left(\frac{\alpha \beta}{\beta+t z}\right) d t=\frac{\alpha \beta}{z} \int_{\alpha}^{\beta} \frac{f(s)}{s^{2}} d s .
$$

Replacing $x$ by $\frac{a b}{b+t \eta(a, b)}$ and $y$ by $\frac{a b}{a+t \eta(b, a)}$ in (5), we obtain

$$
f\left(\frac{2 a b}{a+b}\right) \leq \frac{\bar{h}(x, y)}{2}\left(f\left[\frac{a b}{b+t \eta(a, b)}\right]+f\left(\frac{a b}{a+t \eta(b, a)}\right)\right)
$$

for all $t \in[0,1]$, implying

$$
\begin{aligned}
& \int_{0}^{1} f\left(\frac{2 a b}{a+b}\right) d t \\
& \quad \leq \frac{\bar{h}(x, y)}{2}\left[\int_{0}^{1} f\left(\frac{a b}{b+\operatorname{t\eta }(a, b)}\right) d t+\int_{0}^{1} f\left(\frac{a b}{a+t \eta(b, a)}\right) d t\right] .
\end{aligned}
$$

Using (6), we get

$$
f\left(\frac{2 a b}{a+b}\right) \leq \frac{\bar{h}(x, y)}{2}\left[\frac{a b}{\eta(a, b)} \int_{a}^{b} \frac{f(x)}{x^{2}} d x-\frac{a b}{\eta(b, a)} \int_{a}^{b} \frac{f(x)}{x^{2}} d x\right]
$$




$$
=\frac{a b \bar{h}(x, y)}{\eta(a, b)} \int_{a}^{b} \frac{f(x)}{x^{2}} d x .
$$

For fixed $a, b \in K, f$ satisfies

$$
f\left(\frac{a b}{b+t \eta(a, b)}\right) \leq h(a, b, t) f(b)+(1-h(a, b, t)) f(a),
$$

for all $t \in[0,1]$. Applying (6), we have

$$
\begin{aligned}
\int_{0}^{1} f\left(\frac{a b}{b+t \eta(a, b)}\right) d t \leq & \int_{0}^{1} h(x, y, t) f(b) d t \\
& +\int_{0}^{1}(1-h(x, y, t)) f(a) d t \\
= & f(a) \int_{0}^{1} h(x, y, t) d t \\
& +f(b) \int_{0}^{1}(1-h(x, y, t)) d t \\
= & \bar{h}(x, y) \frac{f(a)+f(b)}{2},
\end{aligned}
$$

i.e.,

$$
\frac{a b}{\eta(a, b)} \int_{a}^{b} \frac{f(x)}{x^{2}} d x \leq \bar{h}(x, y) \frac{f(a)+f(b)}{2} .
$$

Hence (7) and (8), we get

$$
f\left(\frac{2 a b}{a+b}\right) \leq \frac{a b \bar{h}(x, y)}{\eta(a, b)} \int_{a}^{b} \frac{f(x)}{x^{2}} d x \leq[\bar{h}(x, y)]^{2} \frac{f(a)+f(b)}{2} .
$$

This completes the proof.

Theorem 8. For any set $K=\subset \mathbb{R} \backslash\{0\}$, let $\eta: K \times K \rightarrow \mathbb{R}$ be a vector valued function. Let $K_{\eta} \subset \mathbb{R} \backslash\{0\}$ be a $H I$ set associated with $h$ defined by

$$
y+h(x, y, t) \eta(x, y)= \begin{cases}y, & \text { if } t=0 \\ x, & \text { if } t=1\end{cases}
$$

for all $x, y \in K$. Let $\eta$ be antisymmetric on $K$. Let $f: K \rightarrow \mathbb{R}$ be a $H I$ function associated with $h$ on $\operatorname{Int}(K)$ with respect to the direction $\eta \in \mathbb{R} \backslash\{0\}$. Let $f^{\prime} \in L[a, b]$, then the following inequality hold:

$$
\bar{h}(a, b) \frac{f(a)+f(b)}{2}-\frac{a b}{\eta(b, a)} \int_{a}^{b} \frac{f(x)}{x^{2}} d x
$$




$$
=\frac{a b \bar{h}(a, b) \eta(b, a)}{2} \int_{0}^{1} \frac{1-2 t}{(a+t \eta(b, a))^{2}} f^{\prime}\left(\frac{a b}{a+t \eta(b, a)}\right) d t .
$$

Proof. Assuming $x=\frac{a b}{a+h(a, b, t) \eta(b, a)}=\frac{a b}{a+t \bar{h}(a, b) \eta(b, a)}$, we get

$$
t=\frac{a b-a x}{\bar{h}(a, b) \eta(b, a) x} \Rightarrow 1-2 t=\frac{\bar{h}(a, b) \eta(b, a)+2 a}{\bar{h}(a, b) \eta(b, a)}-\frac{2 a b}{\bar{h}(a, b) \eta(b, a) x}
$$

and

$$
d t=-\frac{(a+t \bar{h}(a, b) \eta(b, a))^{2}}{a b \bar{h}(a, b) \eta(b, a)} d x .
$$

Since $a+h(a, b, 1) \eta(b, a)=a+\bar{h}(a, b) \eta(b, a)=b$ and $t: 0 \rightarrow 1$, we have $x: b \rightarrow \frac{a b}{a+\bar{h}(a, b) \eta(b, a)}=a$. Thus

$$
\begin{aligned}
& \frac{a b \eta(b, a)}{2} \int_{0}^{1} \frac{1-2 t}{(a+h(a, b, t) \eta(b, a))^{2}} f^{\prime}\left(\frac{a b}{a+h(a, b, t) \eta(b, a)}\right) d t \\
& =\frac{1}{2} \int_{a}^{b}\left(\frac{\bar{h}(a, b) \eta(b, a)+2 a}{\bar{h}(a, b) \eta(b, a)}-\frac{2 a b}{\bar{h}(a, b) \eta(b, a) x}\right) f^{\prime}(x) d x \\
& =\frac{b-a}{2 \bar{h}(a, b) \eta(b, a)}[f(a)+f(b)]-\frac{a b}{\bar{h}(a, b) \eta(b, a)} \int_{a}^{b} \frac{f(x)}{x^{2}} d x \\
& =\frac{f(a)+f(b)}{2}-\frac{a b}{\bar{h}(a, b) \eta(b, a)} \int_{a}^{b} \frac{f(x)}{x^{2}} d x,
\end{aligned}
$$

since $b-a=\bar{h}(a, b) \eta(b, a)$. Thus

$$
\begin{gathered}
\frac{a b \bar{h}(a, b) \eta(b, a)}{2} \int_{0}^{1} \frac{1-2 t}{(a+h(a, b, t) \eta(b, a))^{2}} f^{\prime}\left(\frac{a b}{a+h(a, b, t) \eta(b, a)}\right) d t \\
=\bar{h}(a, b) \frac{f(a)+f(b)}{2}-\frac{a b}{\eta(b, a)} \int_{a}^{b} \frac{f(x)}{x^{2}} d x .
\end{gathered}
$$

This completes the proof.

\section{References}

[1] G.D. Anderson, M.K. Vamanamurthy, and M. Vuorinen, Generalized convexity and inequalities, Journal of Mathematical Analysis and Applications, 335 (2007), 1294-1308.

[2] M.A. Hanson, On sufficiency of the Kuhn-Tucker conditions, Journal of Mathematical Analysis and Applications, 80 (1981), 545-550. 
[3] I. Insan, Hermite-Hadamard type inequalities for harmonically convex functions, Hacettepe Journal of Mathematics and Statistics, 43(6) (2014), 935-942.

[4] S.N. Mishra, P.K. Das, and G. C. Nayak, Some results associated with HermiteHadamard type inequalities in invariant harmonic convex set, Global Journal of Pure and Applied Mathematics (2017), Accepted. 